\title{
Open Tray Impression Technique Using the Direct Pick-Up Coping: A Case Report
}

\author{
Debbabi Imen ${ }^{1 *}$, Harzallah Belhassen ${ }^{2}$, Ben khelifa Mohamed ${ }^{3}$ and Cherif Mounir ${ }^{4}$ \\ ${ }^{1}$ Department of Fixed Prosthodontics, faculty of dental medicine, Tunisia \\ ${ }^{2}$ Department of Fixed Prosthodontics, Faculty of dental medicine, Tunisia \\ ${ }^{3}$ Dental surgery department, Hospital University Center of Dental Medicine Monastir, Tunisia \\ ${ }^{4}$ Department of Fixed Prosthodontics, Faculty of dental medicine, Tunisiasssssss
}

Received: 眥August 13, 2018; Published: 制 August 17, 2018

*Corresponding author: Debbabi Imen, Department of Fixed Prosthodontics, Dental Clinic of Monastir, Tunisia

\begin{abstract}
Dental implants have become a quite successful method for restoration of fully and partially edentulous patients. Impression procedure becomes exacting in implantology as compared to fixed partial denture because of lack of periodontal ligament fibers in implants. Traditionally, there are two different implant impression techniques for transferring the impression copings from the implant to the impression. The transfer technique uses tapered copings and a closed tray to make an impression. Conversely, the pick-up impression uses square copings and an open tray (a tray with an opening), allowing the coronal ends of the impression coping screw to be exposed. This article describes the Pick-up implant impression procedure that is inexpensive, clean, and easy to perform with materials commonly found in a restorative dental practice and deals with its advantages and limitations.
\end{abstract}

Keywords: Implant Impression; Implant Direct Impression; Open Tray - Pick Up Impression Coping

\section{Introduction}

The success of implant-supported restorations relies on how well the hard and soft tissue information is transferred to the laboratory [1,2]. The critical aspect is to record the threedimensional orientation of the implant as it is present intraorally, other than reproducing fine surface detail for successful implant prosthodontic treatment [3-5]. The development of impression techniques to accurately record implant position has become more complicated and challenging. Several impression techniques have been suggested to obtain a master cast that will ensure the passive fit of prosthesis on implants [6]. In implant dentistry, the two traditional ways of making an impression are the closed tray and the open tray technique. Both impression techniques have their advantages and disadvantages [7]. A recent systematic review revealed that the open tray impression technique is more accurate than the closed tray impression technique [8,9]. It has been shown that the pickup type impression coping is the more accurate type of impression as errors occur on removal and replacement of the transfer type impression copings, especially in the occlusion-gingival direction $[10,11]$. Characteristics of the
Pickup type impression coping are that they are removed from the mouth together with the set impression. They require access to the retaining screw to allow release of the screw prior to removal of the impression coping-impression assembly, the analogues are attached to the impression copings while they are embedded in the impression tray. A custom tray with access to the impression coping screws is required [12].

\section{Clinical Case}

\section{Open Tray Impression Procedure}

A patient consulted the Department of Fixed Prosthodontics at the Dental Clinic of Monastir to replace his bilateral terminal maxillary edentulism by implant-supported fixed prostheses. The patient had a sufficient and rectilinear mouth opening. We decided to make him five maxillary implants. To record the spatial position of the implants, we have chosen "Open tray Impression technique "using the direct pick-up copings. In the first time, the healing screws were removed (Figure 1). Then, the square impression copings were placed into the implants (Figures 2 \& 3). The guide screws were 
tightened using the screwdriver. Retroalveolar X-rays were taken along the long axis of the implant to ensure that the impression copings were seated completely into the hex of the implants (Figure 1). The tray was perforated in the regions where implants were placed to provide access for the pick-up copings (Figure 4). The impression tray was coated with manufacturer recommended impression adhesive 5 minutes before each impression was made. Tray adhesive was applied thinly and evenly over the inner surface of each tray and extended approximately $3 \mathrm{~mm}$ onto the outer surface of the tray along periphery. The adhesive was allowed to dry for 15 minutes before impression (Figure 4). The tray was removed from the mouth and two sections of boxing wax (Dentsply Intl) were adapted over the open-ings in the tray and sealed to the tray using a hot instrument (Figures 5 \& 6).
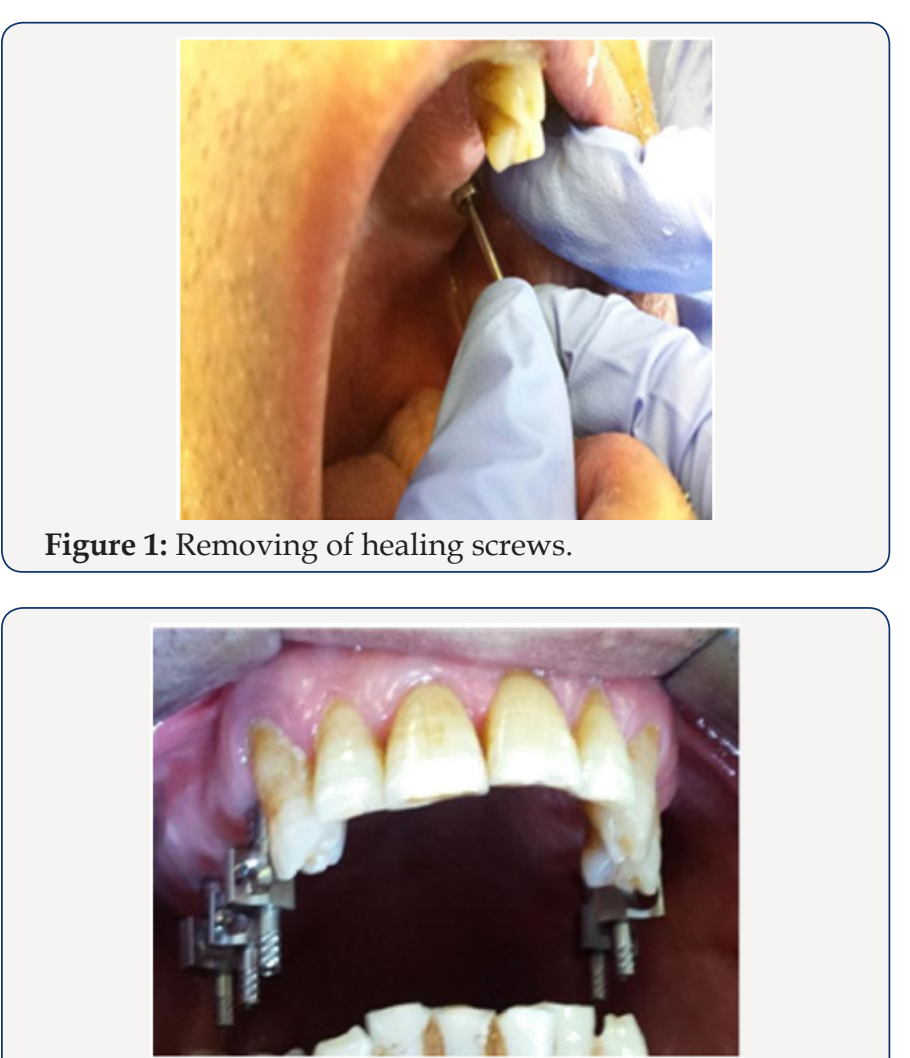

Figure 2: Impression copings were connected to each implant after removing of healing screws.

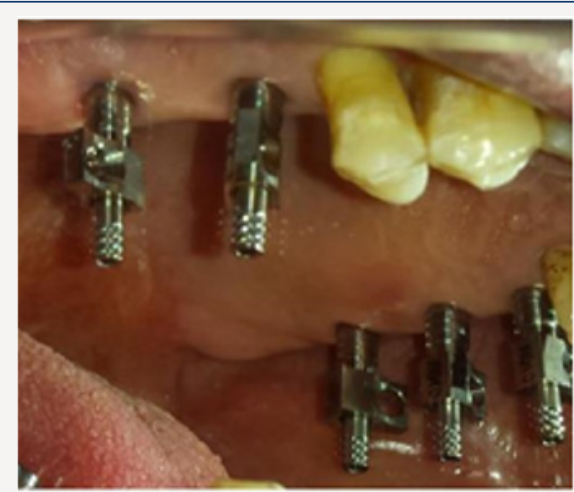

Figure 3: Impression copings were connected to each implant after removing of healing screws.
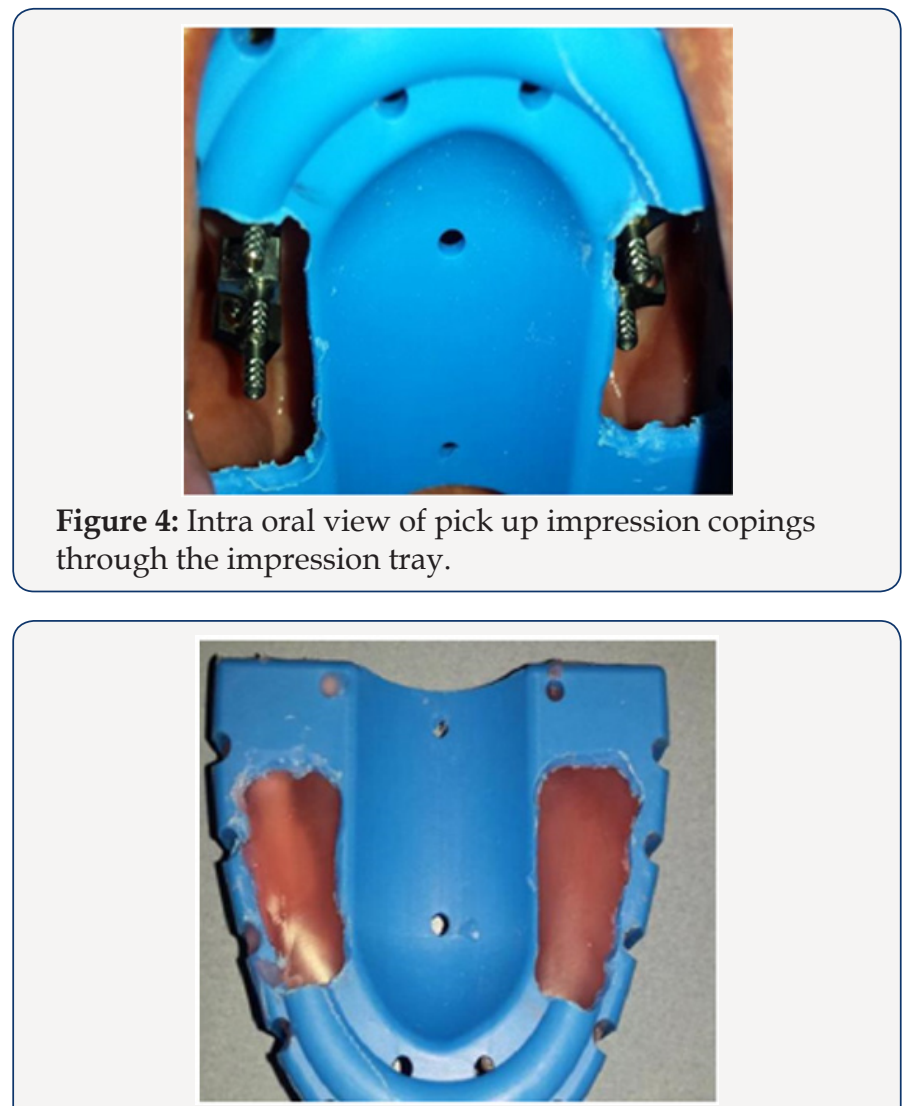

Figure 5: two sections of boxing wax (Dentsply Intl) were adapted over the open-ings in the tray.

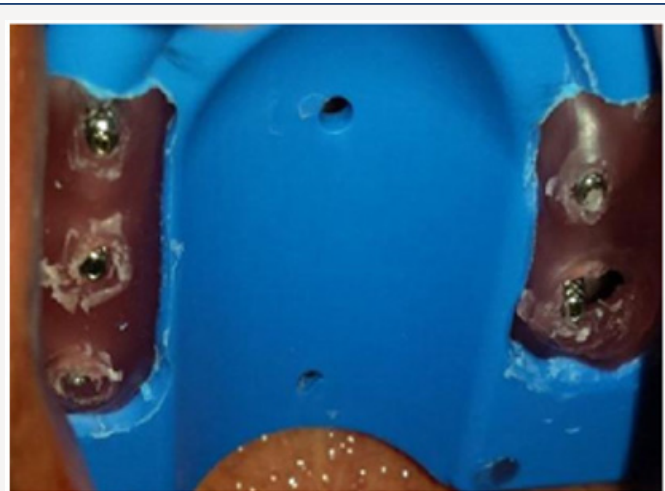

Figure 6: two sections of boxing wax (Dentsply Intl) were adapted over the open-ings in the tray.

Other open-tray impression protocols recommend wiping off the excess impression material that extrudes through the hole in the tray with a gloved finger or injecting impression plaster through the hole(s) in the tray to fill the remaining void. This may be messy to accomplish. Additionally, the powder on some gloves may inhibit the polymerization of vinyl polysiloxane (VPS) impression materials Wax placed over the hole in the tray prevents contact of the impression material with the gloves [13]. The heavy consistency polyvinylsiloxane impression material was loaded inside the impression tray and light consistency polyvinylsiloxane impression material was meticulously syringed around the impression copings to ensure complete coverage of the 
copings (Figure 7). The screwdriver was used to loosen the guide screw within the impression post (Figure 8). The impression was taken out from the patient's mouth. The corresponding analog was selected and the impression post was placed into the implant analog and the guide screw was tightened by using the screwdriver (Figure 9). The healing abutments were replaced immediately to prevent soft tissue collapse over the implant (Figure 10). Before screwing in, implant analog should be placed in line with the grooves of impression coping to achieve accurate passively fitting prosthesis. The impression was now ready to be used to create a model (Figure11).

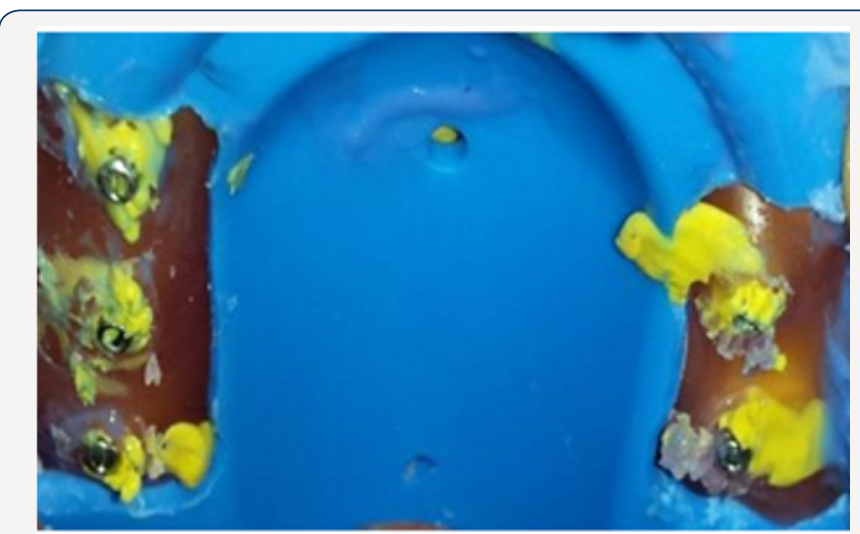

Figure 7: Open tray Impression.

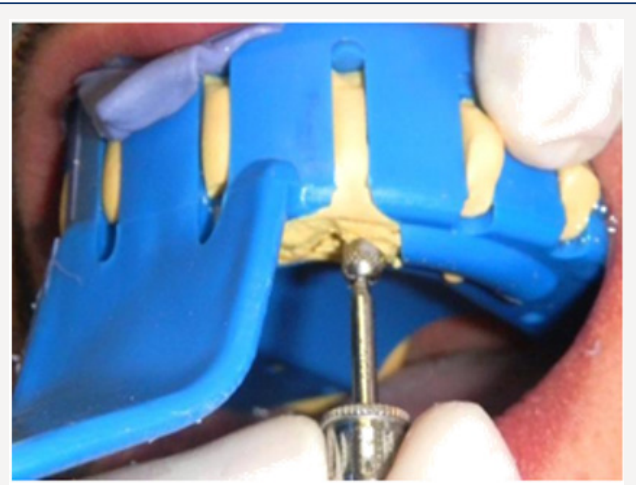

Figure 8: The copings screws are unscrewed to be removed along with the impression.

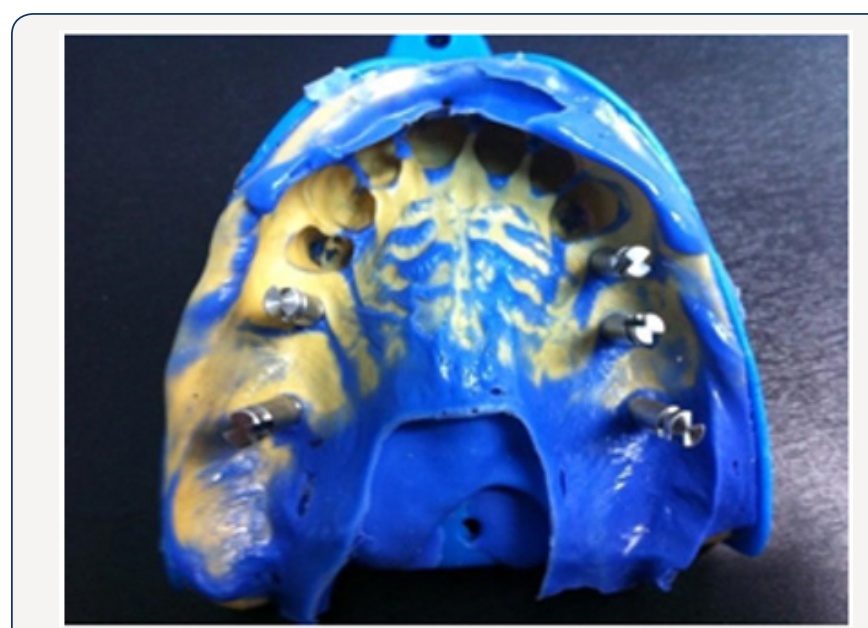

Figure 9: Impression with coping analog assembly.

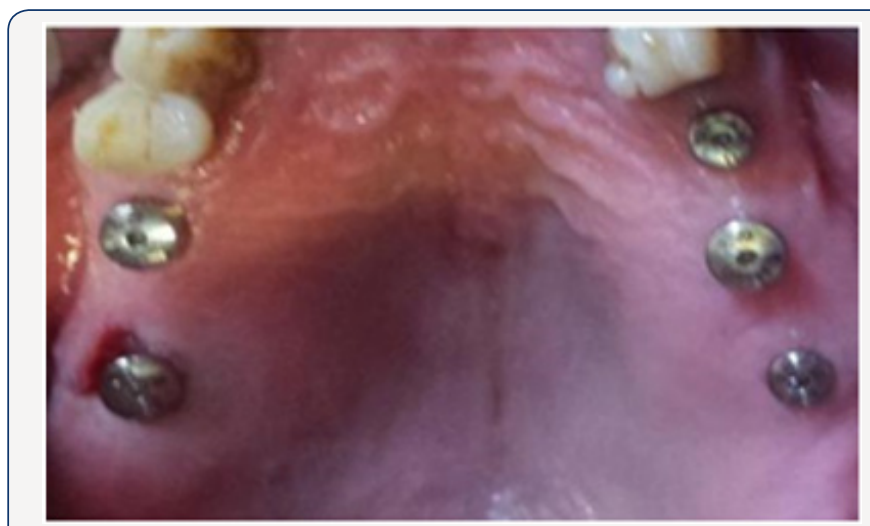

Figure 10: The healing abutments were replaced immediately.

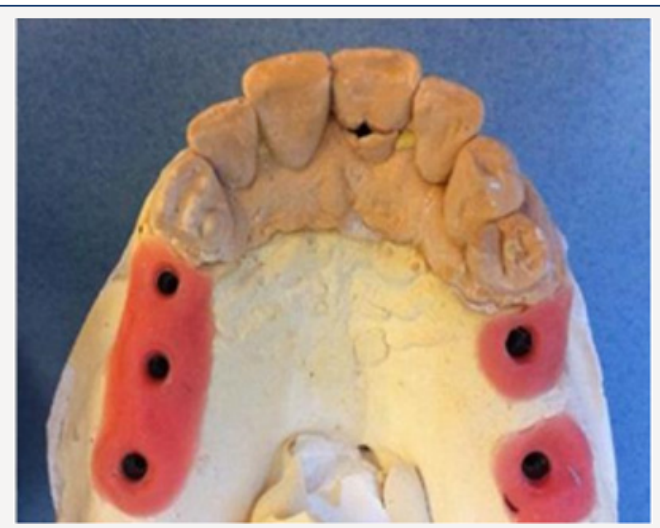

Figure 11: Occlusal view of hard and soft tissue cast recovered from impression.

\section{Discussion}

In direct technique, the impression coping lies within the impression. Advantages of direct technique are minimization of dimensional changes in impression upon removal from patient's mouth, no need to screw in coping again as it comes along with the impression and useful in patients with angulated implants [14]. When impressions are required for multi implant restorations the precision of the impression is even more critical. This is because frameworks will be constructed from the master cast and mis-fit in the frameworks can lead to stress applied to the implants on screwing down the framework [12]. For situations in which there were three or fewer implants, most studies showed no difference between the pick-up and transfer techniques, whereas for situations in which there were 4 or more implants, more studies showed more accurate impressions with the pick-up technique (open tray) than the transfer technique (closed tray) [6]. Fourteen studies compared the accuracy of pick-up and transfer impression techniques $[15,16]$. Of the 14 studies, five showed more accurate impressions with the non-splinted pick-up technique [17], two with the transfer technique, and three showed no difference between them $[8,18]$.

Closed tray (transfer, indirect) technique using tapered impression copings is an easy and simple technique ideal for single implant impressions and multiple parallel implants in patients with limited mouth opening [14]. When there is limited mouth opening 
they can be used as there may not be sufficient space for access to the screws retaining pick up type impression copings with the impression in place and in patients with an exaggerated gag reflex, when the impression has to be removed as quickly as possible [12]. The clinical situations which indicate the use of the closed tray technique are when the patient has limited inter arch space, tendency to gag, or if it is too difficult to access an implant in the posterior region of the mouth [10].

Polyether and VPS were the recommended materials for the implant impressions. Results indicated that the 2-step VPS impression was significantly less accurate than the 1-step putty and light-body VPS combination impression, the mediumbody VPS monophase impression, and the medium-body polyether monophase impression [5].

\section{Conclusion}

Several impression techniques have been advocated for implant impressions to obtain a definitive cast. Different impression techniques have some advantages and some limitations, but selection of technique depends upon operator choice and various clinical situations. Open tray (pick-up, direct) technique using square impression copings is more accurate method usually preferred in multiple implants with different angulations.

\section{References}

1. Assif D, Marshak B, Schmidt A (1996) Accuracy of implant impression techniques. Int J Oral Maxillofac Implants 11(2): 216-222.

2. Daoudi MF, Setchell DJ, Searson LJ (2004) An evaluation of three implant level impression techniques for single tooth implants. Eur J Prosthodont Restor Dent 12(1): 9-14.

3. Barrett MG, De RijkWG, Burgess JO (1993) The accuracy of six impression techniques for osseointegrated implants. J Prosthodont 2(2): 75-82.

4. Wee AG, Aquilino SA, Schneider RL (1999) Strategies to achieve fit in implant prosthodontics: a review of the literature. Int J Prosthodont 12(2): 167-178.

5. DR Prithviraj, Malesh L Pujari , Pooja Garg DP Shruthi (2011) Accuracy of the implant impression obtained from different impression materials and techniques: review. J Clin Exp Dent 3(2): 106-111.
6. Lorenzoni M, Pertl C, Penkner K, Polansky R, Sedaj B, et al. (2000) Comparison of the transfer precision of three different impression materials in combination with transfer caps for the Frialit-2 system. J Oral Rehabil 27(7): 629-638.

7. Sumit Bhansali, Sonal Priya Bhansali, Priyanka Negi (2017) A Modified open tray implant impression technique for limited mouth opening. The Journal of Prosthetic Dentistry 118(1): 116-117.

8. Lee H, So JS, Hochstedler JL, Ercoli C (2008) The accuracy of implant impressions: a systematic review. J Prosthet Dent 100(4): 285-291.

9. Moreira AHJ, Rodrigues NF, Pinho ACM, Fonseca JC, Vilaça JL (2015) Accuracy comparison of implant impression techniques: a systematic review. Clin Implant Dent Relat Res 17: e751-e764.

10. Liou AD, Nicholls JI, Yuodelis RA, Brudvik JS (1993) Accuracy of replacing three tapered transfer impression copings in two elastomeric impression materials. Int J Prosthodont 6(4): 377-383.

11. Spector MR, Donovan TE, Nicholls JI (1990) An evaluation of impression techniques for osseointegrated implants. J Prosthet Dent 63(4): 444447.

12.W Chee, S Jivraj (2006) Impression techniques for implant dentistry. British Dental Journal 201(7): 429-432.

13. Richard J, Windhorn Thomas R, Gunnell (2016) A simple open-tray implant impression technique. The Journal of Prosthetic Dentistry 96(3): 220-221.

14. Shweta Singh, Alok Kumar (2016) Implant Impression Techniques in Dentistry. Journal of Dental Sciences and Oral Rehabilitation 7(3): 137141.

15. Inturregui JA, Aquilino SA, Ryther JS, Lund PS (1993) Evaluation of three impression techniques for osseointegrated oral implants. J Prosthet Dent 69(5): 503-509.

16. Phillips KM, Nicholls JI, Ma T, Rubenstein J (1994) The accuracy of three implant impression techniques: a three-dimensional analysis. Int J Oral Maxillofac Implants 9: 533-540.

17. Cabral LM, Guedes CG (2007) Comparative analysis of 4 impression techniques for implants. Implant Dent 16 (2): 187-194.

18. Debbabi I, Saafi J, Harzallah B, Cherif M (2018) Implant Impression: What about Splinted Impression Copings? Mod App Dent Oral Health 2(3): 1-4.
This work is licensed under Creative Commons Attribution 4.0 License

To Submit Your Article Click Here: Submit Article
DOI: 10.32474/MADOHC.2018.03.000155

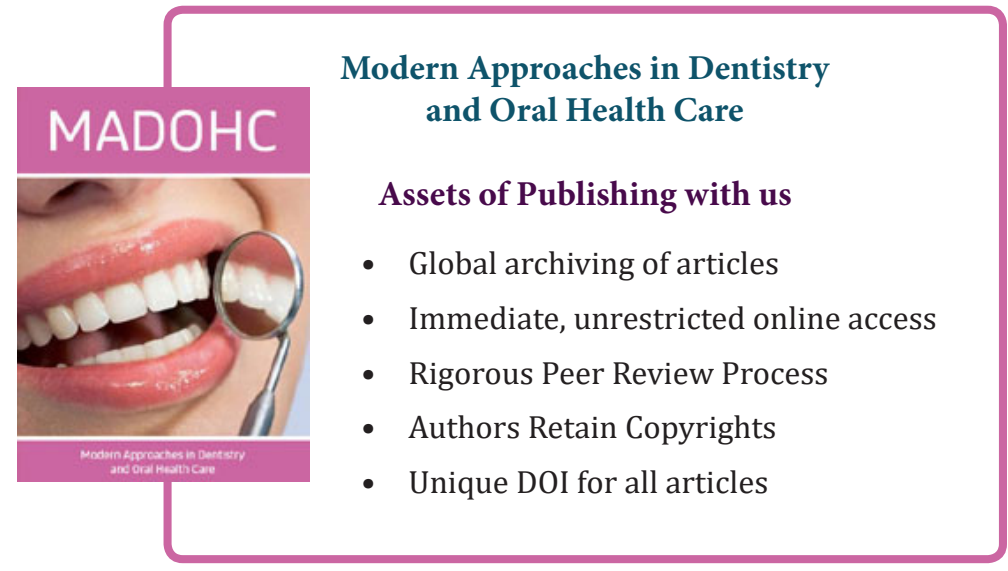

